\title{
Transatlantica
}

Revue d'études américaines. American Studies Journal

1 | 2016

Modernist Revolutions: American Poetry and the

Paradigm of the New

\section{Passion Patchwork : l'étoffe de l'Amérique}

\section{Adélaïde Desnoé}

\section{(2) OpenEdition}

Journals

Édition électronique

URL : https://journals.openedition.org/transatlantica/8272

DOI : $10.4000 /$ transatlantica.8272

ISSN : 1765-2766

Éditeur

Association française d'Etudes Américaines (AFEA)

Référence électronique

Adélaïde Desnoé, «Passion Patchwork : l'étoffe de l'Amérique », Transatlantica [En ligne], 1 | 2016, mis en ligne le 17 février 2017, consulté le 31 janvier 2023. URL : http://journals.openedition.org/

transatlantica/8272 ; DOI : https://doi.org/10.4000/transatlantica.8272

Ce document a été généré automatiquement le 31 janvier 2023.

\section{(c) (1) $(9)$}

Creative Commons - Attribution - Pas d'Utilisation Commerciale - Pas de Modification 4.0 International - CC BY-NC-ND 4.0

https://creativecommons.org/licenses/by-nc-nd/4.0/ 


\section{Passion Patchwork : l'étoffe de l'Amérique}

\section{Adélaïde Desnoé}

1 Lors de la première exposition de la saison 2016-2017 d'Art Hop Polis, le rendez-vous culturel « inter-maisons » de la Cité internationale, les quilts de la collection de CharlesEdouard de Broin ont fait l'objet de deux expositions successives, exposées aux cimaises de la galerie de la Fondation des Etats-Unis. Ces expositions inédites sont un hommage au patchwork américain, dont une formidable sélection a été proposée aux visiteurs.

2 Géraldine Chouard, Professeure à l'Université Paris-Dauphine, Directrice du groupe de recherches HIMAN (Histoire des Images en Amérique du Nord), au sein du LARCA (UMR 8225) à l'Université Paris-Diderot, spécialiste de patchwork et commissaire des expositions, et Charles-Edouard de Broin, collectionneur de quilts et passionné d'art populaire partagent ici leur passion commune. Retour sur une pratique très florissante aux Etats-Unis, depuis toujours, et pourtant encore relativement peu connue en France. 


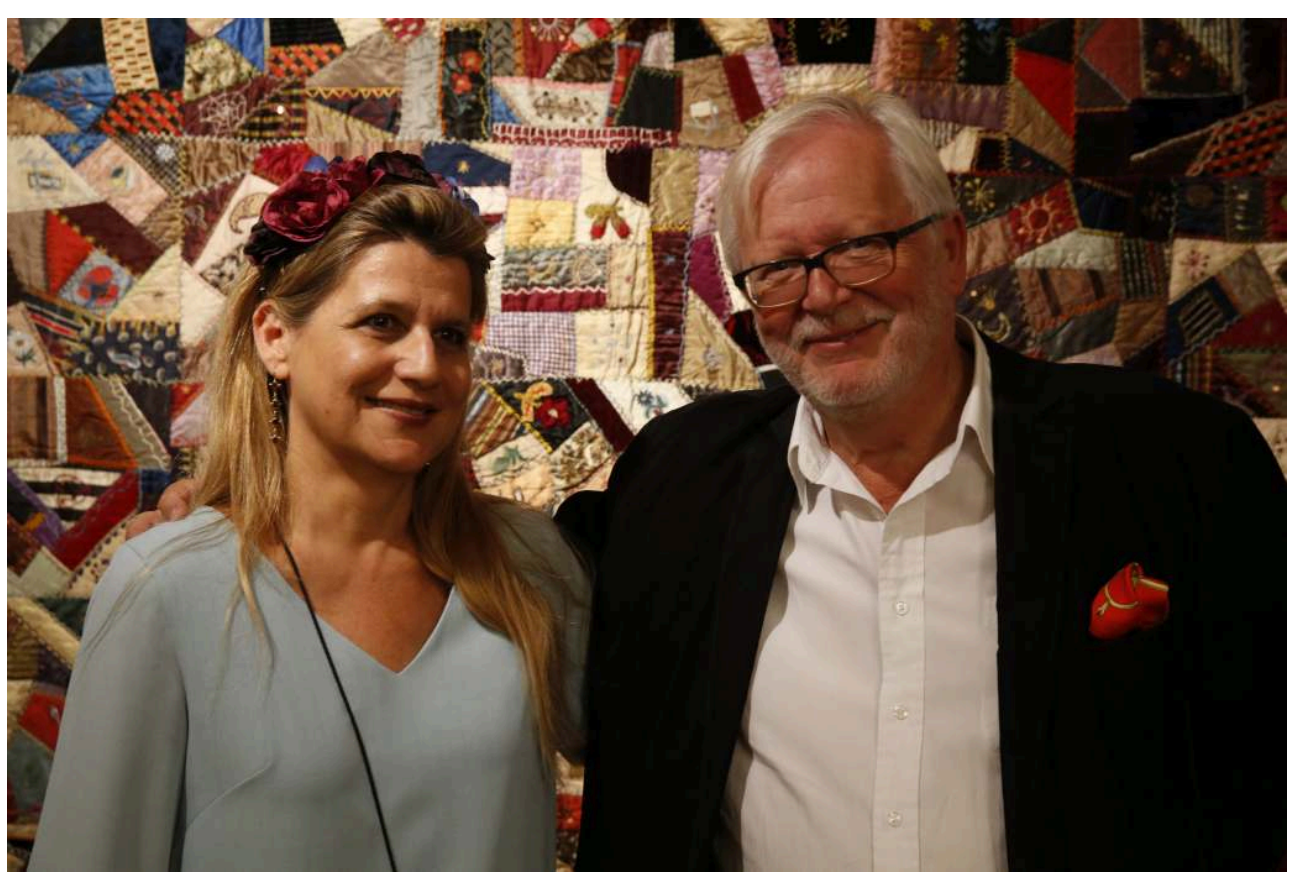

Fig. 1 : Géraldine Chouard et Charles-Edouard de Broin, Fondation des Etats-Unis, Vernissage de l'exposition « Le fil de la désobéissance : les Crazy Quilts », 5 novembre 2016

(C) Philippe Lissac

Adélaïde Desnoé [A. D.] : Question de lexique pour commencer, quelle est votre définition du quilt?

Ch-E. de Broin : Le terme « quilt » fait référence au procédé de matelassage du tissu par des points de couture qui le rendent plus solide et plus confortable. Conçu à l'origine comme un simple objet utilitaire, le quilt est peu à peu devenu un objet d'art, qui s'affiche aujourd'hui aux cimaises des musées comme un tableau.

G. Chouard : Le patchwork (littéralement : patch-work) désigne la pratique, c'est-àdire l'art de mettre ensemble des morceaux de tissu. La prononciation du mot «quilt» est importante : il ne s'agit pas de «kilt», comme on l'entend parfois, qui renvoie à une autre culture. En français, on préfère utiliser le terme « patchwork », ce qui simplifie les choses.

\section{A. D.: D'où est venu votre intérêt pour les quilts?}

Ch-E. de Broin : Comme une rencontre amoureuse. C'était en 1985 à Houston, au Texas, lors d'un séjour inhérent à mon métier de géophysicien. Une précédente expatriation avait suscité chez moi une vive curiosité pour l'art populaire des EtatsUnis. Et je m'intéressais par ailleurs, depuis longtemps, aux ouvrages textiles, ayant collectionné et brodé moi-même des abécédaires. Et c'est là que j'ai découvert ces assemblages bariolés, ordonnés autant que fantaisistes, composés à partir de tissus de récupération, presque toujours chargés d'histoire. Il faut rappeler que le quilt est parfois le seul bien qu'emportaient les pionniers au moment de quitter une Europe famélique ou en guerre pour rejoindre le Nouveau Monde. Ces objets d'art populaire si simples, si utiles et si beaux m'ont immédiatement séduit. 


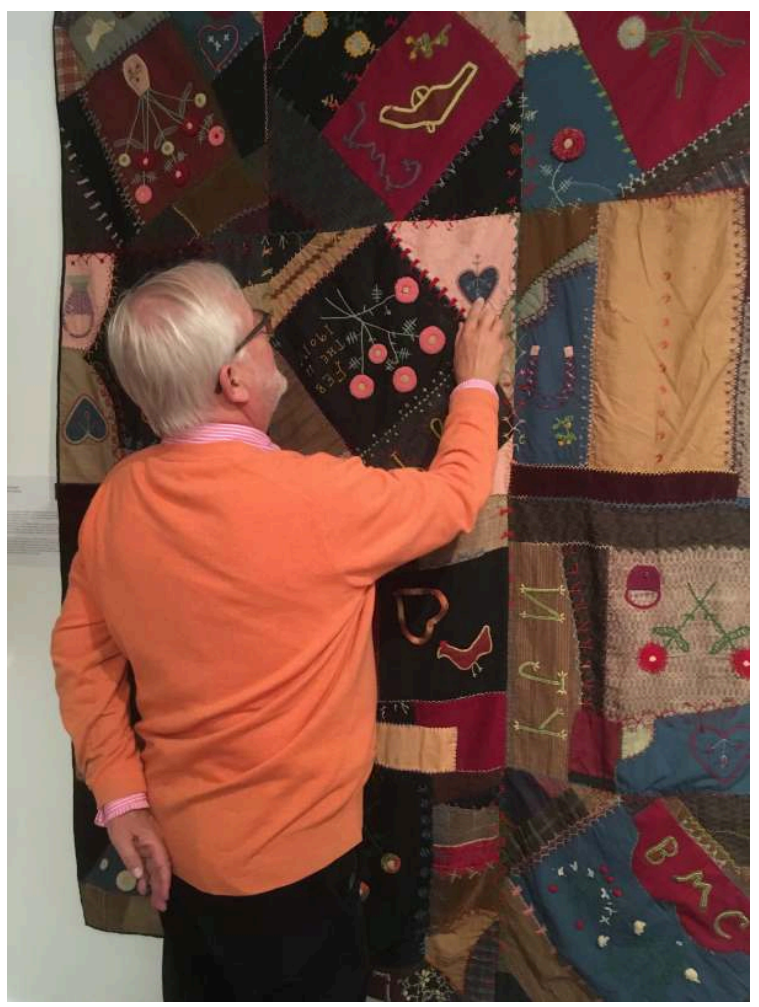

Fig. 2 : Charles-Edouard de Broin, devant « Crazy Quilt, From Mollie», New York Co, 1901 (158 × 210 $\mathrm{cm})$

(c) Adélaïde Desnoé

G. Chouard : De mon côté, mon intérêt, qui remonte déjà à plus d'une vingtaine d'années, vient de la littérature. Du texte, je suis passée à l'image, puis de l'image à la chose.

Dans la fiction américaine, il est souvent question de patchwork. Par exemple, dans un roman d'Eudora Welty, Losing Battles (1970), l'intrigue est centrée autour d'un certain quilt au nom de Delectable Mountains (modèle assez rare, dont l'exposition comporte un exemplaire). 


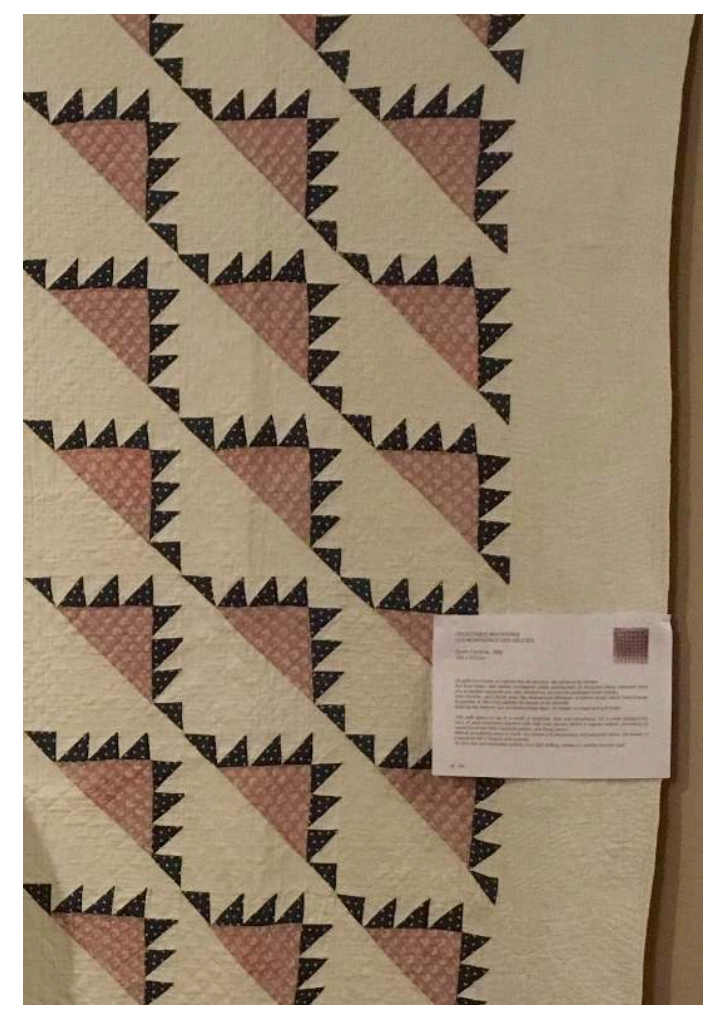

Fig. 3 : «Delectable Mountains », South Carolina 1890 (detail), $(193$ x 210 cm)

(C) Géraldine Chouard

J'ai donc souhaité en savoir davantage. Mon premier contact avec le patchwork américain est lié à la consulation d'encyclopédies, de livres illustrés, d'ouvrages d'histoire. Je cherchais à retrouver visuellement le patchwork. En lisant cette vaste littérature, j'ai alors compris que la pratique américaine avait un lien très fort avec la vie sociale et culturelle des Etats-Unis, qu'elle était très différente de la tradition du patchwork français qui est importante, certes, mais plutôt décorative. Je n'ai depuis plus lâché le fil.

Ch-E. de Broin : Il est vrai que c'est une passion qui ne vous lâche pas! Cela fait maintenant plus de trente ans que je suis collectionneur de quilts. Mon goût a beaucoup évolué au fil du temps. Si c'est la qualité graphique de cet art textile qui a d'abord attiré mon regard, leur contenu historique a vite pris le relai, venant ajouter à leur valeur esthétique. Car chaque quilt a une histoire (individuelle, collective, nationale), qu'il faut constituer, ou reconstituer. J'ai monté cette collection en l'accompagnant d'un catalogue raisonné où chaque quilt est répertorié avec autant d'informations qu'il m'a été possible de rassembler: date, auteur, nom du modèle, circonstances de l'exécution. Quand un quilt ne possède aucun de ces éléments, il est parfois possible de le dater grâce à son modèle («pattern»), mais aussi grâce à certains tissus, suivant le titre d'un ouvrage d'une experte du patchwork, Barbara Brackman, Clues in the Calico: A Guide to Identifying and Dating Antique Quilts (1997). On finit par voir tout quilt comme une énigme à résoudre. 


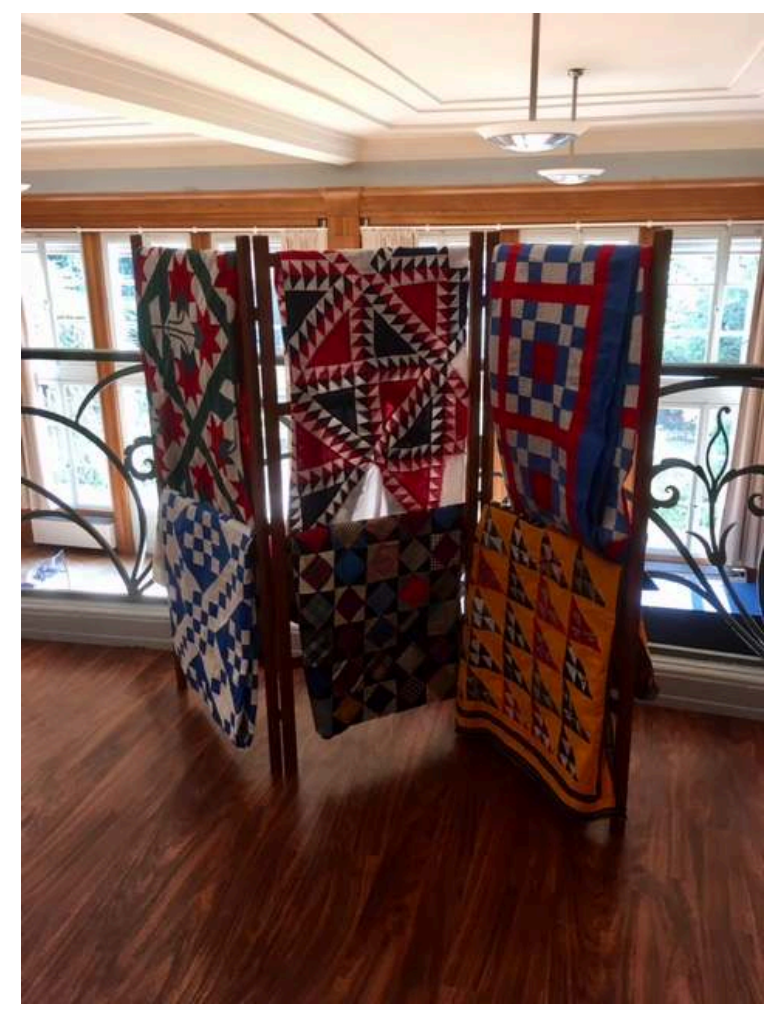

Fig. 4 : Quelques spécimens de la collection de Charles-Edouard de Broin

(c) Géraldine Chouard

A. D. : Voyez-vous les quilts comme des objets utilitaires ou comme des œuvres d'art?

Ch-E. de Broin : Il m'est impossible désormais, en partie pour les raisons que je viens d'indiquer, de faire la distinction. Et c'est précisément cette combinaison qui m'intéresse. Un quilt rustique, ou en très mauvais état, peut être une œuvre d'art. Tout dépend de la manière de le voir. 


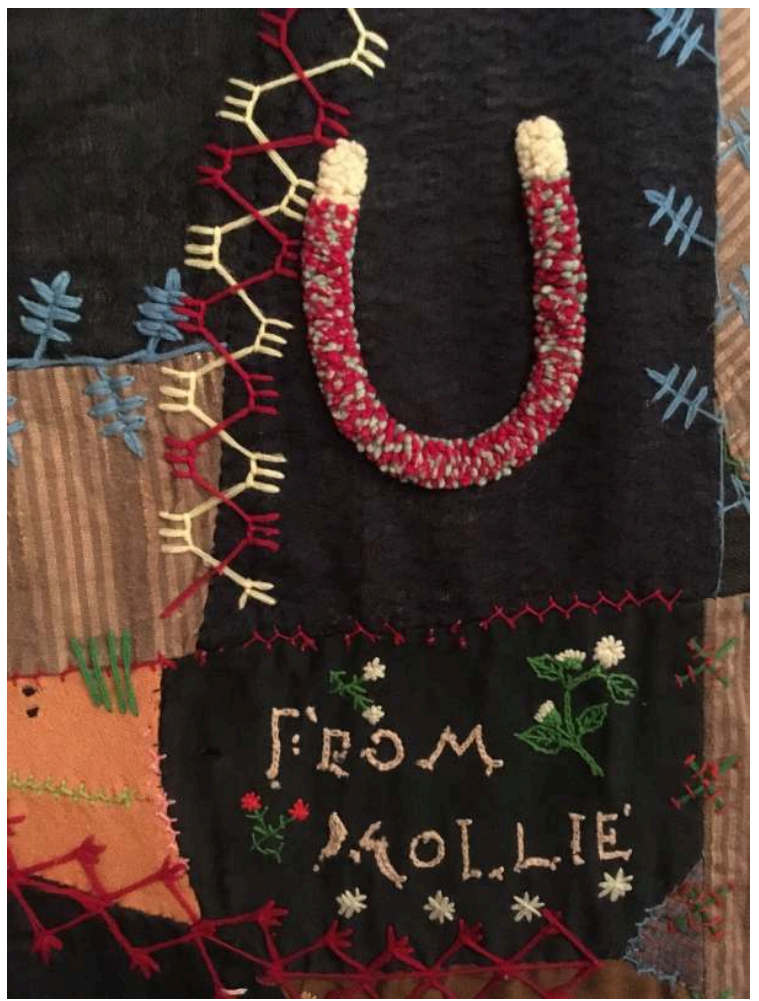

Fig. 5 : «Crazy Quilt: from Mollie», New York, 1901 (détail), (158 x $210 \mathrm{~cm})$

(c) Géraldine Chouard

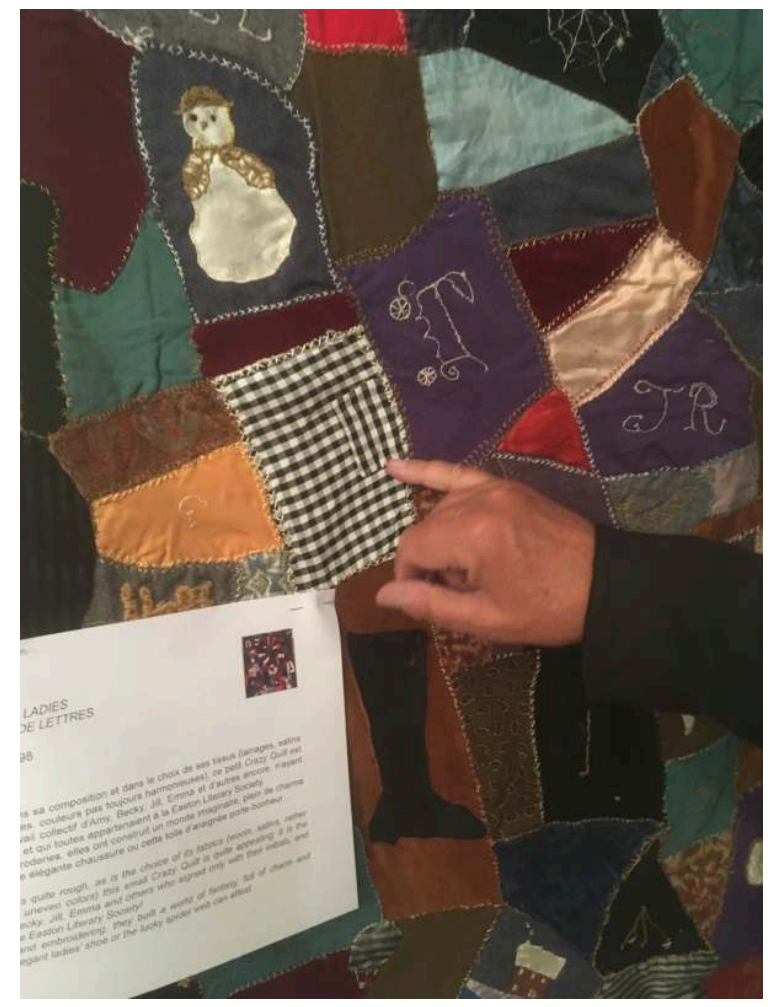

Fig. 6 : « Crazy Quilt, Literary Ladies », New York, 1898 (détail), (100 x 116 cm)

(c) Géraldine Chouard 
$A D$ : Quel est le lien spécifique entre l'Amérique et le patchwork?

G. Chouard : C'est une vaste question! Cette pratique de seconde main, commence dès la période coloniale et scande l'avancée de la nation jusqu'à la période contemporaine, sans interruption. Métaphore géographique de l'Amérique dans tous ses états, le patchwork a été et reste une occasion de la découvrir, quilt après quilt, à chaque étape de son histoire, sous toutes ses coutures.

J'ai eu d'abord du patchwork une approche globale, n'ayant pas idée, au départ, qu'il y avait tant de variétés de quilts, que chaque tendance correspondait à des aires géographiques, à des communautés spécifiques, ou encore à des projets politiques. Ainsi, par exemple, au nord-est, où se sont installés les premiers puritains, les quilts sont souvent réalisés dans des couleurs sobres, suivant des motifs classiques (maison, étoile, compas). En Pennsylvanie, les formes sont épurées et les constructions géométriques. La région de Lancaster est le fief des Amish, dont les créations aux couleurs fortes et tranchées sont proches de l'abstraction contemporaine (Rothko, Albers, Newman), à l'instar de celui qui est présenté dans l'exposition, dans de magnifiques tonalités de pourpre et d'indigo.

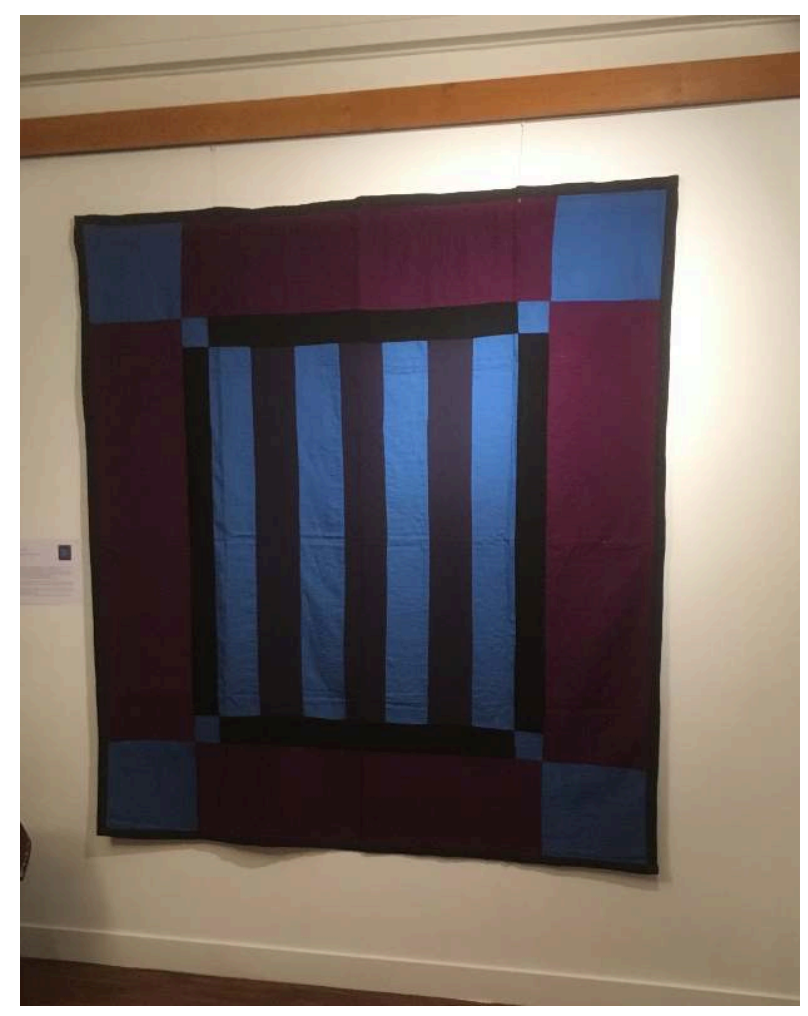

Fig. 7 : « Bars, Amish, Pennsylvanie, Lancaster County, c. 1930. (180 x $192 \mathrm{~cm})$

\section{(C) Charles-Edouard de Broin}

La fonction mémorielle du patchwork américain est également particulièrement intéressante. Il existe un modèle pour chaque type d'occasion, de la naissance à la mort, du « crib quilt» (« patchwork de berceau ») au « mourning quilt» («patchwork de deuil ») en passant par le «wedding quilt (patchwork de mariage), pour ne citer que quelques classiques du répertoire, parmi tant d'autres. 
$\mathrm{Au}$ plan collectif, la coutume est de préserver dans la fibre textile les souvenirs du passé pour leur assurer diffusion et durée : toutes les grandes étapes de la destinée des Etats-Unis sont représentées (naissance de la nation, avancée de la frontière, Guerre de Sécession, abolition de l'esclavage, lutte pour les droits civiques, élections présidentielles, entre autres). La réalisation de quilts conçus en groupe et destinés à être montrés dans des lieux publics donnait ainsi une visibilité aux moments-clés de l'histoire.

Signe des temps, il existe aujourd'hui des $9 / 11$ Quilts sur lesquels figurent le nom des victimes et des messages de soutien de la nation tels que «Remember Always » ou United After all ", sur fond de symboles de New York (notamment les tours jumelles). Ces œuvres de mémoire sont aujourd'hui exposées dans des casernes de pompiers ou dans le hall du récent 9/11 Memorial Museum, à New York.

Ch-E. de Broin : Quand j'ai appris le mode de fabrication des quilts - par les femmes, souvent en groupe, mettant en commun leurs talents d'aiguille pour célébrer un événement dans leur famille, leur communauté villageoise et paroissiale, leur Etat ou leur pays naissant -, j'y ai lu à livre ouvert la vie de la société américaine rurale et laborieuse des XVIIIème et XIXème siècles, sur la côte Est puis au mythique Far West. Humaine, sociale, religieuse ou politique, l'histoire racontée par les patchworks, que je collectionne et restaure depuis plus de trente ans, est passionnante.

A y regarder de près, ces morceaux de tissus disparates, que de minuscules points relient à des centaines voire des milliers d'autres, s'inscrivent dans un ensemble très organisé, résultat d'innombrables combinaisons entre carrés, rectangles et triangles. Tout patchwork est conçu à partir d'un motif - le pattern - représentation symbolique du quotidien des pionniers américains. Cabane de rondins (avec en son centre la chaleur du foyer), construction de la grange, marches du tribunal, vol d'oies sauvages, arbres, sillons des champs, paniers, fleurs ou étoiles figurant un Etat, toute l'Amérique, elle-même en construction, est là sous nos yeux. 


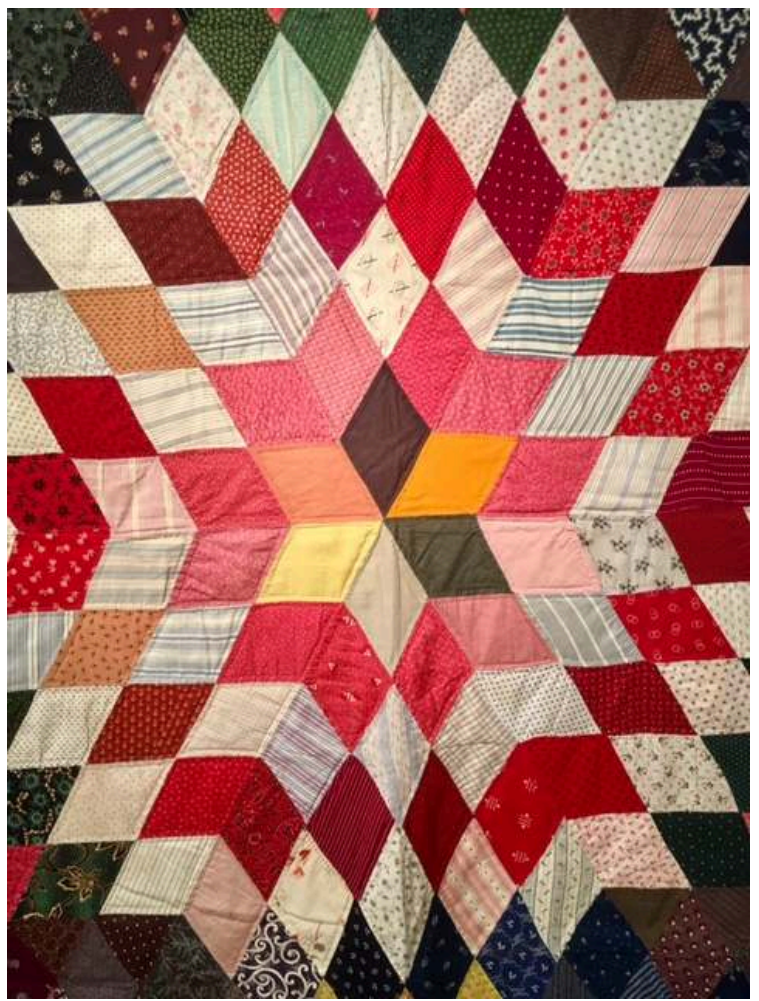

Fig. 8 : « Lemoyne Star, Berkshire Co., Pennsylvanie, 1900-1930 (détail), (196 x $199 \mathrm{~cm}$ )

(C) Charles-Edouard de Broin

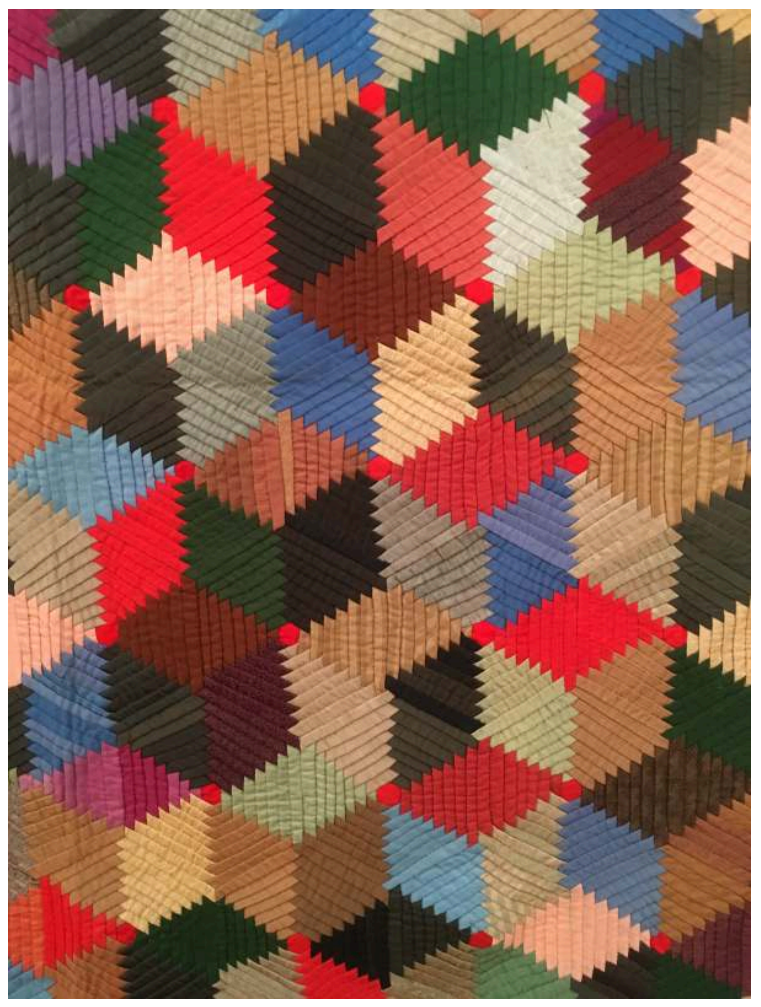

Fig. 9 : « Log Cabin-Pineapple, Connecticut, 1870 (détail), (153 x $163 \mathrm{~cm}$ )

(c) Charles-Edouard de Broin 
Même le "crazy ", un genre fort loin de la rigueur et de la tradition, peut prendre discrètement part au débat public. C'est le cas de « Vignettes » (1900), extraordinaire assemblage de plusieurs milliers de minuscules pièces de soies multicolores, qui fait figurer les portraits du Président William Mc Kinley et du Vice-Président Théodore Roosevelt, élus en 1897, sur des vignettes textiles qu'on trouvait à l'époque dans les paquets de cigarettes. Art de la récupération et expression politique se rejoignent ici de manière exemplaire.

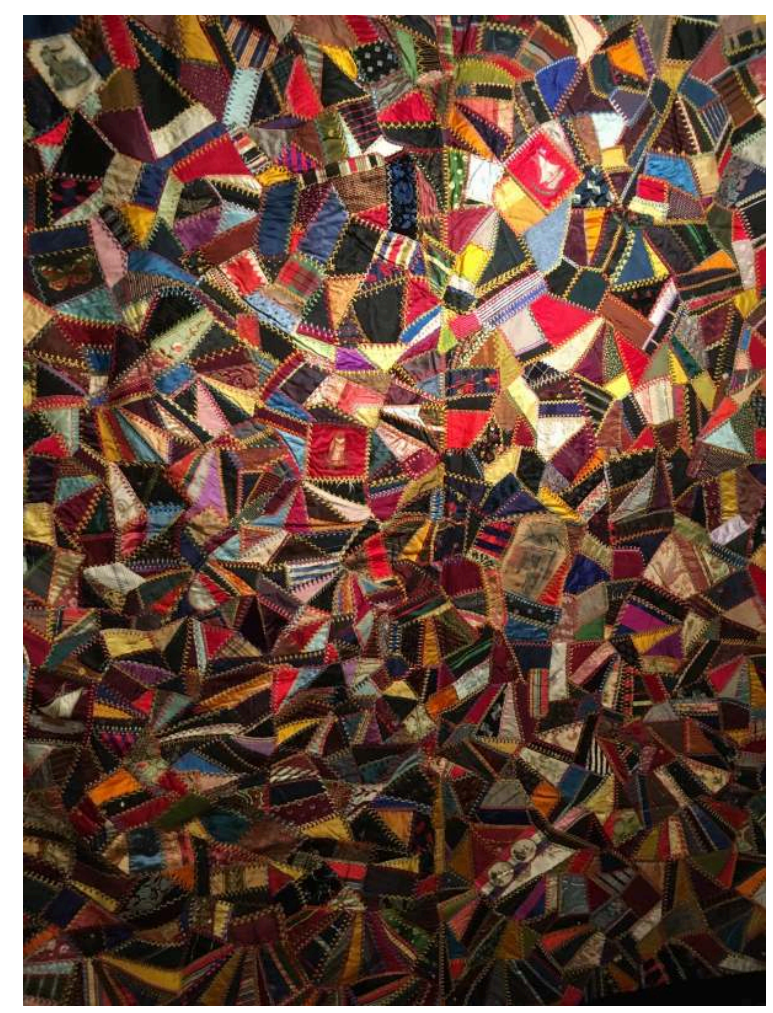

Fig. 10 : « Crazy Quilt , Vignettes », Mrs Bond, Pennsylvanie, 1900, (191 x 204 cm)

(c) Charles-Edouard de Broin 


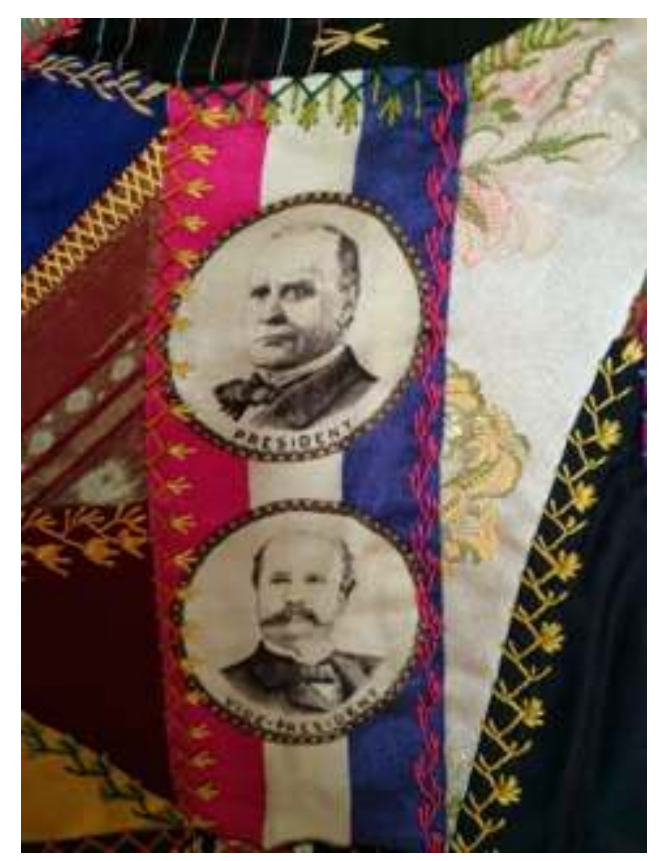

Fig. 11 : détail de «Crazy Quilt, Vignettes », Mrs Bond, Pennsylvanie, 1900, (191 x $204 \mathrm{~cm})$ et

(C) Charles-Edouard de Broin

\section{A.D. : Comment ces expositions ont-t-elle vu le jour? En tant que commissaire, comment vous avez-vous procédé au choix des quilts?}

G. Chouard: Le patchwork est souvent affaire de partage de tissus, d'histoires, d'affects. Et de rencontre. Cette exposition ne fait pas exception à la règle.

J'ai eu l'occasion de travailler sur ce sujet avec Anne Crémieux (qui co-dirige avec Sophie Vasset la Fondation des Etats-Unis), que j'ai en quelque sorte « convertie » au patchwork il y a plus de dix ans! Sa recherche sur les minorités ethniques aux EtatsUnis, notamment sur la communauté afro-américaine, a croisé la mienne, sur les arts textiles. Nous avons réalisé ensemble deux documentaires sur des artistes afroaméricaines contemporaines du patchwork, Riché Richardson. Portrait of the Artist: From Montgomery to Paris (2008) et Gwen Magee, Threads of History (2012), qui ont été montrés en France et aux Etats-Unis.

C'est à l'occasion d'une projection-débat du second documentaire, lors de l'exposition «Art Quilt» à la Fondation Mona Bismarck, en 2013, que j'ai eu la chance de rencontrer Charles-Edouard, expert textile et collectionneur, qui avait accumulé une collection de quilts américains aux multiples motifs, genres et provenances, d'une qualité digne des plus grands musées textiles. Nous avons donc monté ensemble ce projet d'exposition, à l'invitation de la Fondation des Etats-Unis. En fait, sa collection est telle qu'il y avait vraiment l'embarras du choix...

Le plaisir esthétique du quilt étant souvent lié à ses couleurs, le premier regroupement envisagé était d'ordre chromatique, autour du rouge et du bleu en particulier, à l'origine de créations souvent spectaculaires. Mais pour mieux diffuser la connaissance de la pratique, nous avons finalement préféré donner à en voir un large panorama, c'est-à-dire panacher à la fois des modèles classiques du répertoire, souvent géométriques, tels que le «Log Cabin» (cher au collectionneur) ou les 
"Tumbling blocks », mais aussi un Amish, un quilt -américain, ou une pièce historique rare (« Burgoyne Surrounded »).

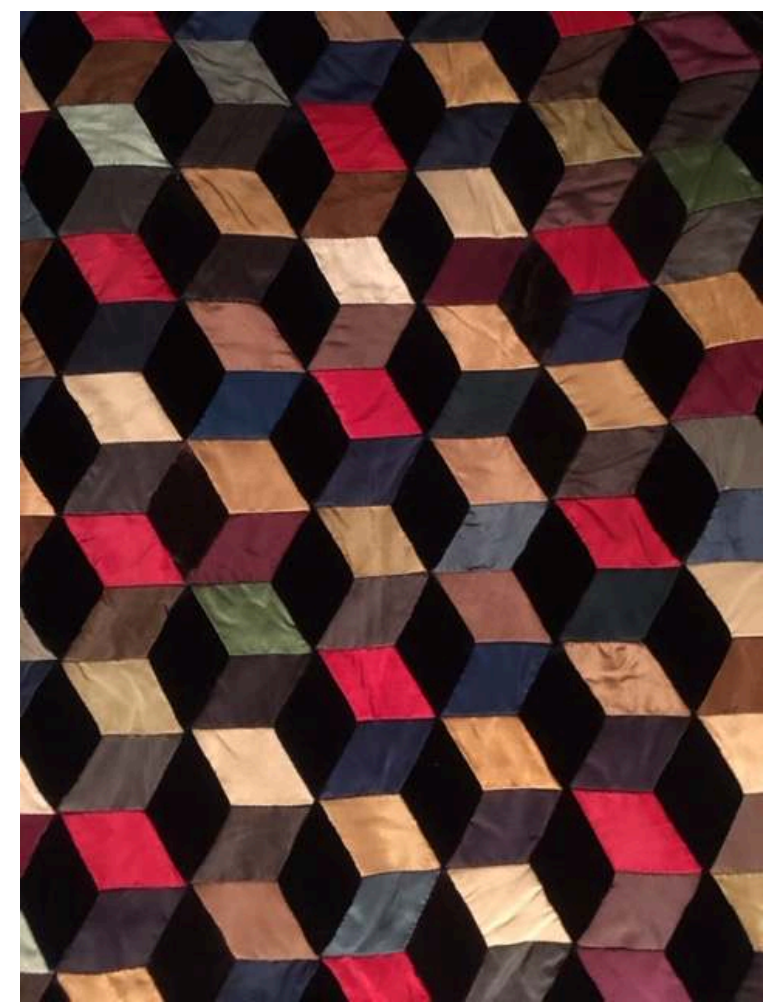

Fig. 12 : «Tumbling Blocks », Connecticut, 1860-90, (détail), (168 x $189 \mathrm{~cm})$

(c) Charles-Edouard de Broin

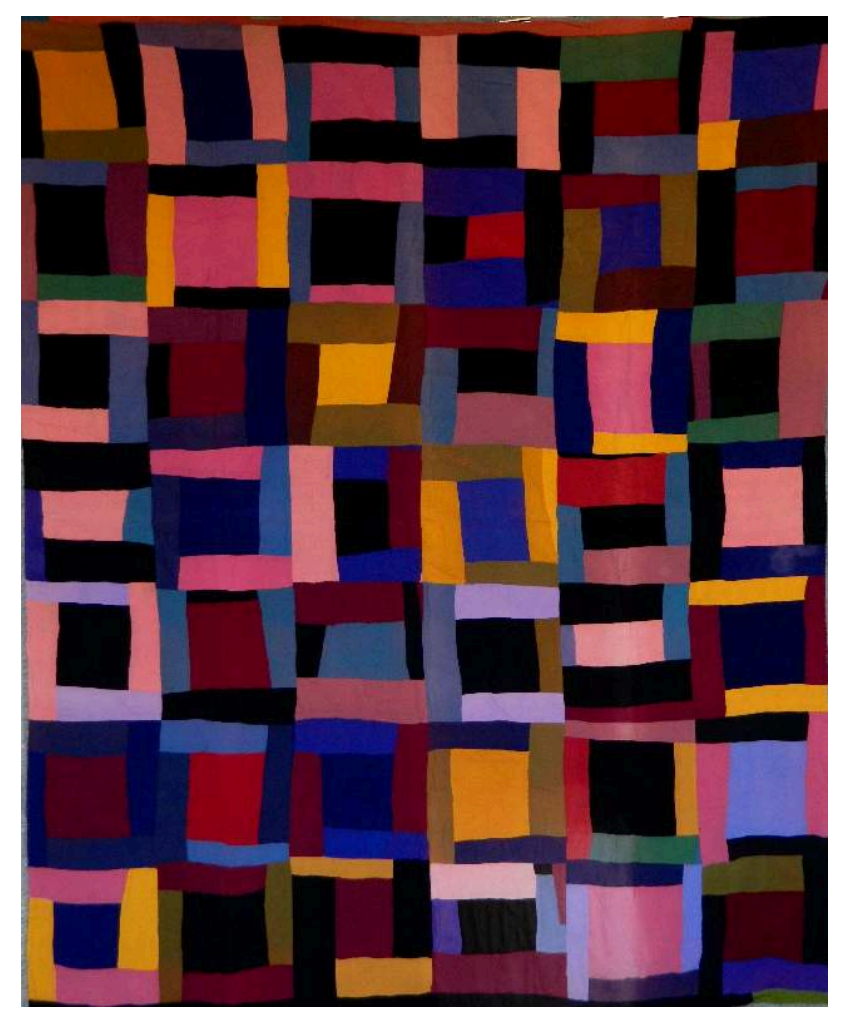


Fig. 13 : «Blocs afro-américains », Virginia, 1940, (180 x 214 cm)

(C) Adélaïde Desnoé

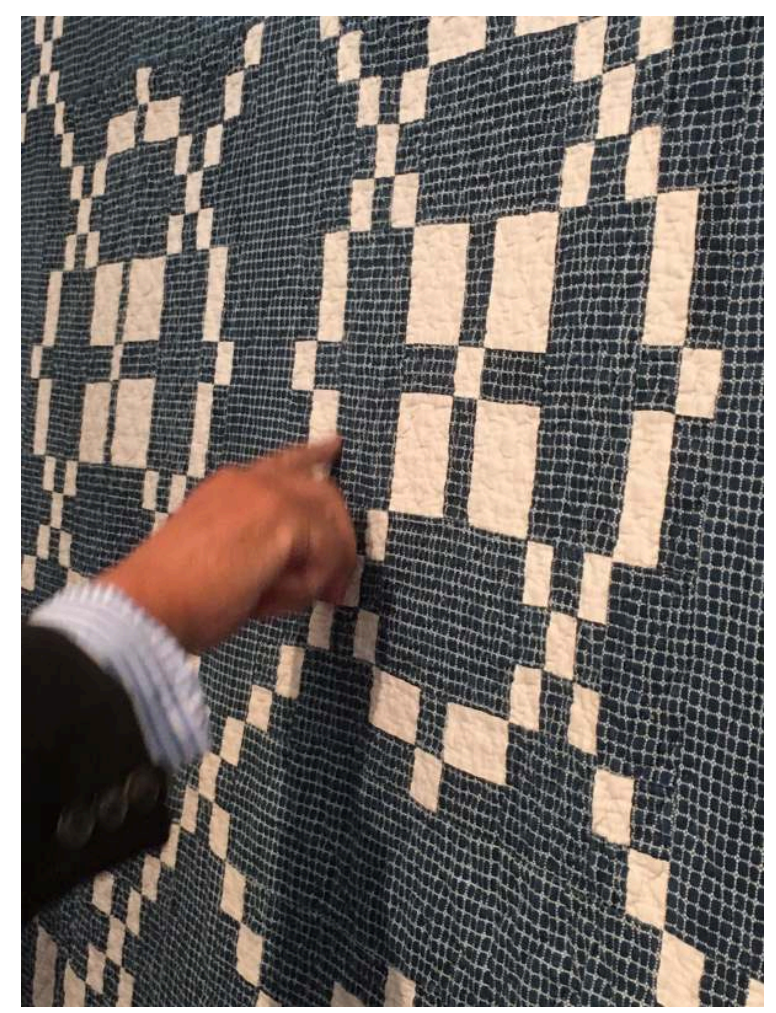

Fig. 14 : « Burgoyne Surrounded », Pennsylvanie, 1910-20 (détail), (190 x $241 \mathrm{~cm})$

(c) Adélaïde Desnoé

Après cette focale large, si l'on peut dire, la seconde exposition proposait de faire le point sur les « Crazy», un genre tout à fait à part, aussi chatoyant que...désobéissant.

A.D : Pourquoi cet intérêt spécifique pour le crazy, justement?

Ch-E. de Broin : Ce qui est «fou» dans ce style de quilt, c'est la libération des femmes qui les ont faits. Le "Crazy» est un moment très particulier qui émerge vers la fin du $19^{\text {ème }}$, dans un milieu victorien très rigide. Au lieu de suivre les patrons (patterns), comme elles l'avaient (presque) toujours fait jusqu'alors, les femmes ont laissé libre cours à leur imagination et se sont mises à assembler couleurs et matières en toute fantaisie. 


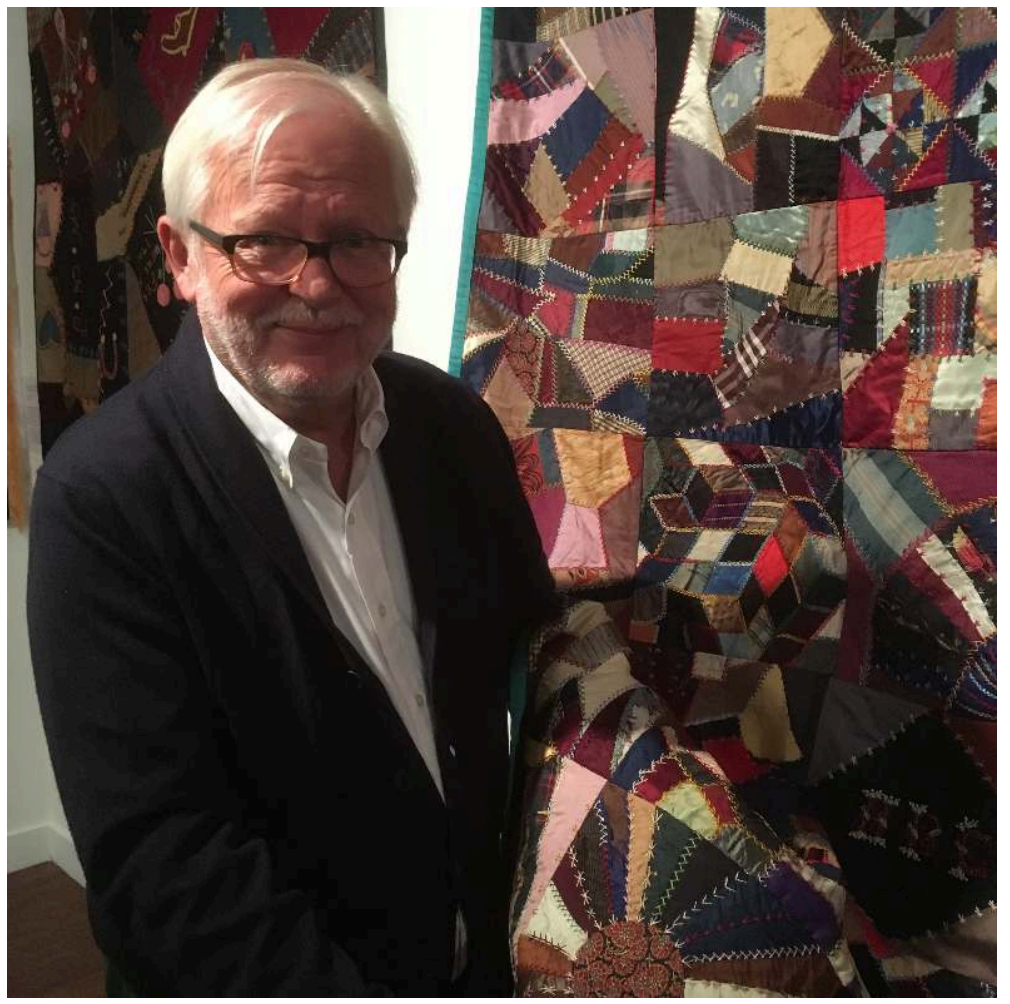

Fig. 15 : «Charles-Edouard de Broin \& Crazy Quilt : Dresden Plate », New York, 1890, (207 x 208 cm)

\section{(c) Géraldine Chouard}

Un des quilts exposé représente une petite fille qui soulève son jupon... A l'époque victorienne, voilà qui a dû faire du raffut! L'audace, l'inventivité, et parfois la désinvolture de ces pièces sont saisissantes. 


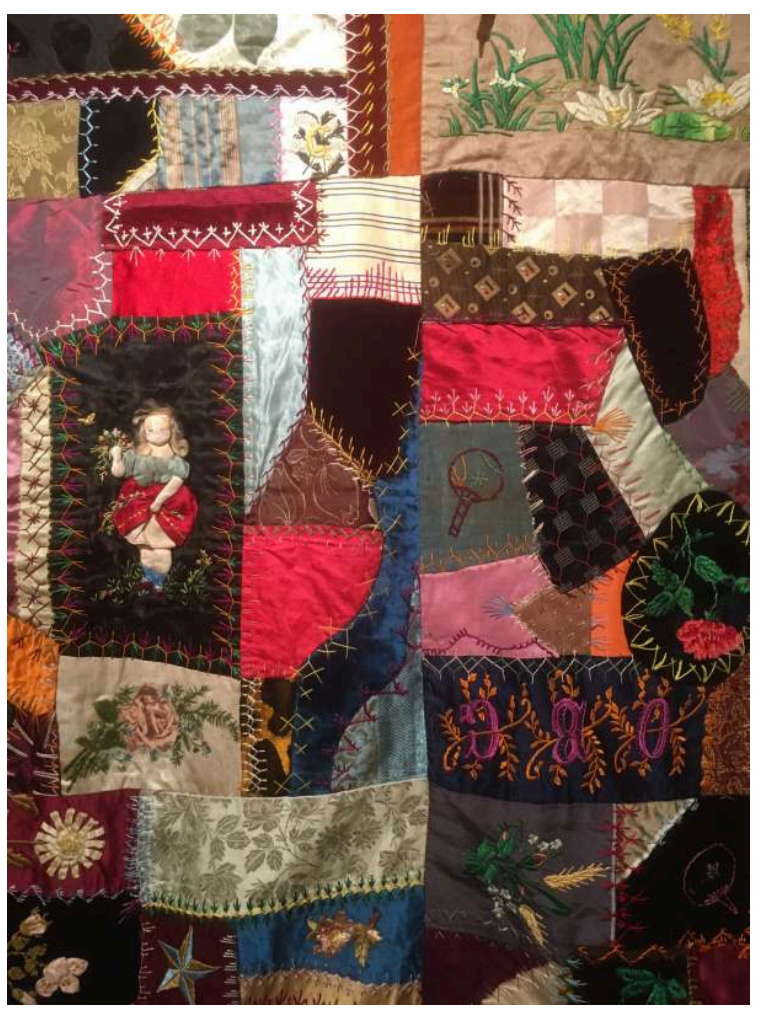

Fig. 16 : «Crazy quilt: With a Doll”, New York, 1890 (détail), (168 x $168 \mathrm{~cm})$

\section{(C) Charles-Edouard de Broin}

En réalité, il y a deux types de «Crazy» distincts, qui sont ici représentés. Les premiers, que l'on trouve surtout dans les milieux aisés de la côte Est, et notamment à New York, visent à montrer les tissus de luxe dont les femmes disposent, ainsi que leur savoir-faire en matière de broderie.

Dans les milieux plus modestes, par exemple dans les campagnes, les femmes suivent cette mode à distance, et recyclent des tissus plus simples (lainages ou cotonnades), créant des compositions d'un charme rustique hors du commun. Elle n'hésitent pas à y ajouter des initiales ou des messages d'une orthographe approximative, ce qui ajoute encore un côté désarmant à ces ouvrages. 


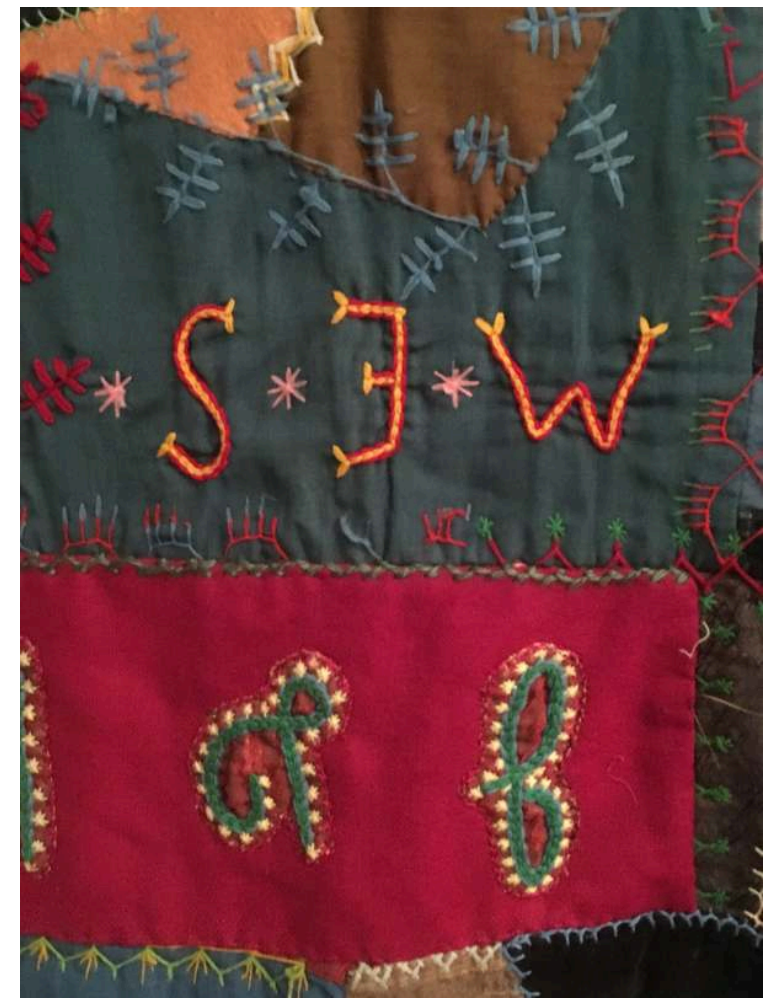

Fig. 17 : «Crazy Quilt: from Mollie », New York, 1901 (détail), $(158 \times 210 \mathrm{~cm})$

(c) Géraldine Chouard

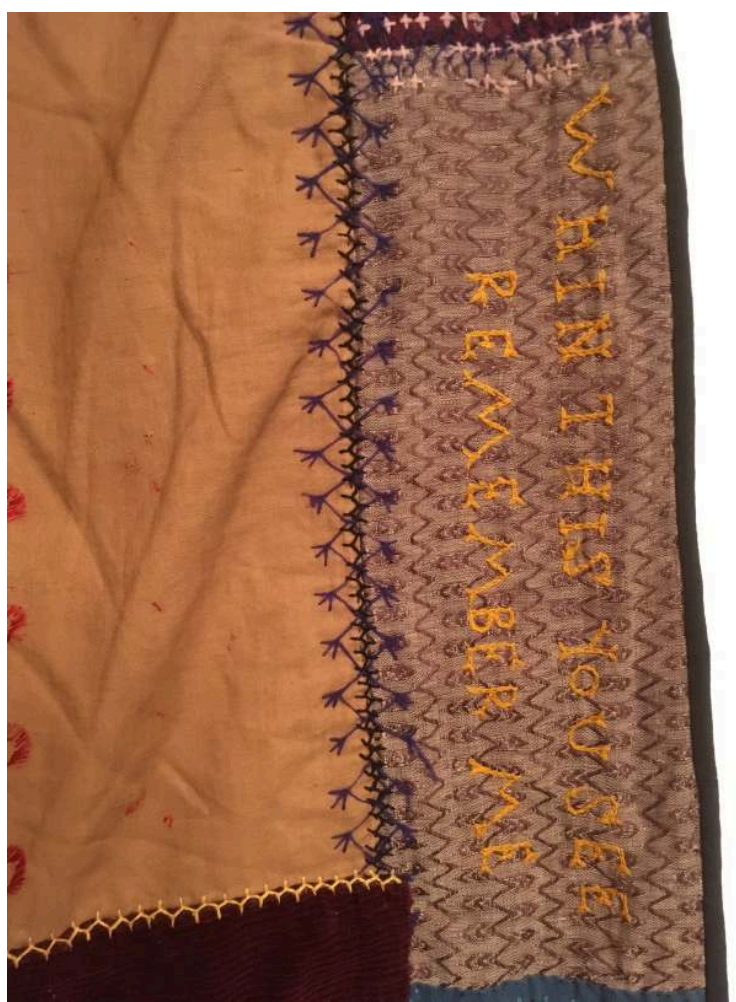


Fig. 18 : «Whin you see me, remember me », « Crazy Quilt: from Mollie », New York, 1901 (détail), $(158 \times 210 \mathrm{~cm})$

\section{(c) Géraldine Chouard}

G. Chouard : Les "Crazy» sont un type de quilts tout à fait à part. Pour comprendre ces pièces hors du commun, il faut bien sûr rappeler l'indépendance dont l'Amérique est $(\mathrm{t})$ issue. L'indépendance, ou même la résistance américaine s'est souvent exprimée à travers le patchwork, notamment au XIXème, par le biais des «Protest quilts ", au service des plus grandes causes de l'histoire nationale, comme l'abolition de l'esclavage, la lutte contre l'alcoolisme ou le droit de vote des femmes. En 1876, lors de l'Exposition universelle de Philadelphie célébrant le centenaire de l'Indépendance, les visiteurs s'extasient devant les arts décoratifs du Pavillon japonais, qui deviennent aussitôt une nouvelle source d'inspiration pour les arts populaires en général et pour le patchwork en particulier.

Avec ses motifs pleins de fantaisie, suggérés par les magazines féminins de l'époque, vite relayés par l'imagination infinie des quilteuses, le "crazy » est un anti-modèle plus qu'un modèle, il leur permet de créer un univers textile où se côtoient étoiles et toupies, éventails et marguerites, chapeaux et bottines, monogrammes et papillons, en toute licence poétique.

Loin des classiques du répertoire, souvent géométriques, le crazy a fait un temps fureur chez les petites mains d'Amérique, renouvelant ainsi leur formidable audace. La mode ne durera que quelques décennies, tombant déjà en désuétude en 1910. C'était une immense chance de pouvoir admirer quelques spécimens de la collection de Charles-Edouard de Broin, et l'effet de chatoyance produit par chaque quilt était comme décuplé par l'ensemble. Un vrai feu d'artifice.

\section{A.D : Qu'en est-il du quilt aujourd'hui ?}

Ch-E. de Broin : C'est un extraordinaire business, avec plus de vingt-ving millions de femmes qui font aujourd'hui du patchwork aux Etats-Unis. La meilleure façon de se rendre compte du phénomène est le succès des foires au patchwork, très nombreux, avec leurs expos-ventes, à la fois de quilts, mais aussi de tissu et tout le matériel permettant de les réaliser, comme par exemple le festival de Houston, qui se tient tous les ans en novembre. Sans oublier la littérature qui accompagne le patchwork (ouvrages, magazines). En dehors des prouesses techniques dont témoignent les femmes (et aussi les quelques hommes) qui poursuivent ces travaux d'aiguille, le patchwork sert de terrain d'expression sociale et politique, d'une manière qui a toujours défini l'histoire américaine et qui continue de le faire. Il n'y a pas d'équivalent dans d'autre société.

G. Chouard : Le quilt continue aujourd'hui à être un vecteur privilégié de la mémoire et à accompagner les grandes causes de la nation. C'est notamment le cas de The Names Quilt (ou The AIDS Memorial Quilt) un projet majeur, datant de 1987, qui réactualisait la tradition du Mourning Quilt (réalisé à l'origine avec les vêtements du défunt). Ce gigantesque patchwork de patchworks conçu en hommage aux personnes décédées du SIDA (dont chaque pièce portrait le nom) fut d'abord exposé à Washington pour alerter les pouvoirs publics sur la gravité de l'épidémie, avant de faire le tour des États-Unis : une manière de redonner corps à ceux qui ne faisaient plus partie du tissu social et de se souvenir d'eux (c'est le double sens du mot remember). The Names Quilt a été partiellement ré-exposé sur le Washington Mall en 
2012, pour les vingt-cinq ans du projet, et il était alors saisissant de voir ce monument textile, telle une pierre tombale "molle», étendue à l'horizontale sur l'esplanade, faire écho aux monuments aux morts ( "memorials») dressés dans toute la ville. Et de constater, encore et toujours, le pouvoir fédérateur du patchwork, capable, en l'occurrence, de rassembler les morts et les vivants. Le Patchwork des Noms a tenté de prendre le relai en France, mais les événements proposés par l'association ne rencontrent auprès du public français qu'un écho mitigé, signe que notre culture n'a guère intégré cette valeur sociale et militante de la pratique.

\section{A.D : Votre impression générale sur ces expositions?}

Ch-E. de Broin : Nous avons été très bien accueillis, Géraldine Chouard et moi-même, par Anne Crémieux et Sophie Vasset (Directrices de la Fondation des Etats-Unis) et par Noëmi Haire-Sievers (responsable culturelle à la FEU) qui ont mis à notre disposition une galerie qui se prêtait fort bien à l'accrochage des quilts et aux panneaux explicatifs dont l'objectif était de permettre à chacun de contextualiser la pratique.

Toute exposition est une réjouissante occasion de raconter l'histoire, petite ou grande, de chaque quilt, un à un, d'en faire admirer l'équilibre, l'harmonie, et aussi d'entrer dans d'infimes détails, dont certains se révèlent parfois en les montrant.

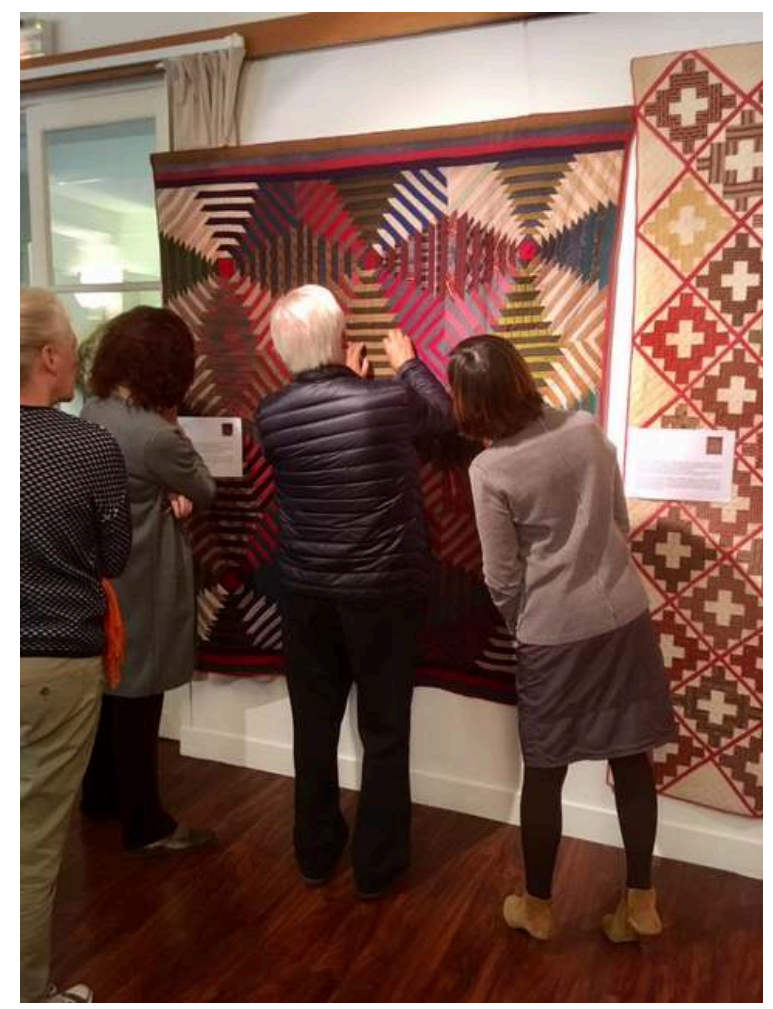

Fig. 19 : « Once Upon a Quilt: I'Amérique comme Patchwork », Fondation des Etats-Unis, octobre 2016

(c) Adélaïde Desnoé 


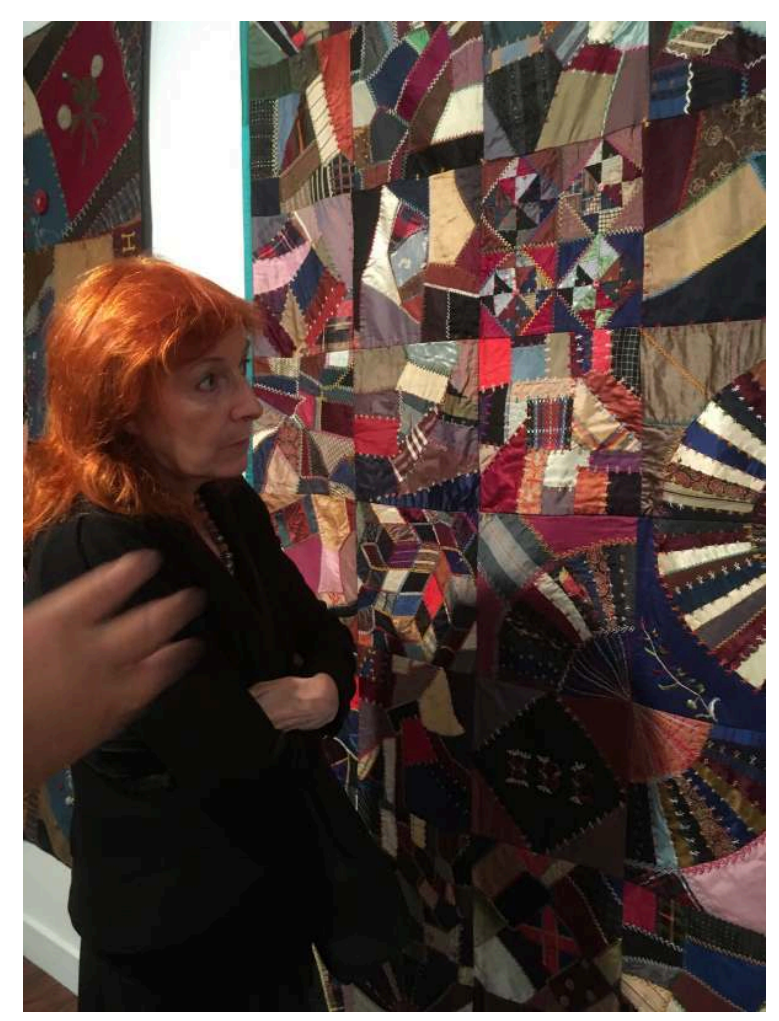

Fig. 20 : «Le fil de la désobéissance : les Crazy quilts », Fondation des Etats-Unis, novembre 2016

\section{(c) Géraldine Chouard}

Le public de la Fondation des Etats-Unis a été fort éclectique et fort réceptif. Les nombreux visiteurs, échangeaient volontiers autour de ces quilts, ce qui a donné lieu à de riches échanges. Une résidente de la Fondation, Marva Dixon, étudiante à l'Institut d'Études Supérieures des Arts, ravie de découvrir sa propre culture ainsi présentée, et appréciée, a proposé de faire le compte-rendu de la première exposition, qui témoigne de sa vision tout à fait originale de l'ensemble.

Le souvenir le plus amusant de cette saison reste celui de ce visiteur, arrivé sur les lieux un peu par hasard, le jour de "Art Hop-Polis ». S'il avait su que c'était une exposition de patchwork, a-t-il dit en partant, il ne serait pas venu. Mais il est resté, médusé par ce qu'il voyait... Ce détour par le patchwork lui avait fait découvrir l'Amérique. Lui aussi! 


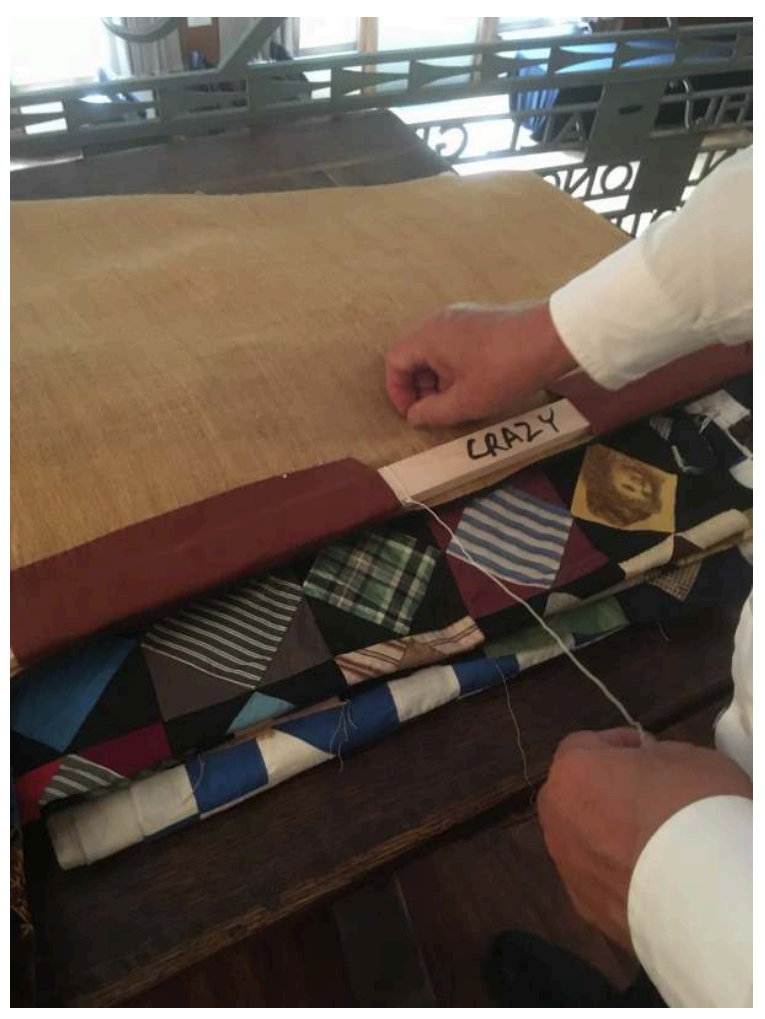

Fig. 21 : Décrochage, Fondation des Etats-Unis, novembre 2016

(C) Géraldine Chouard

\section{RÉSUMÉS}

Entretien avec Géraldine Chouard et Charles-Edouard de Broin de deux expositions à la Fondation des Etats-Unis « Once Upon a Quilt: l'Amérique comme Patchwork » et «Le fil de la désobéissance : les Crazy Quilts » (octobre-novembre 2016)

\section{INDEX}

Thèmes : Trans'Arts

\section{AUTEUR}

\section{ADÉLAÏDE DESNOÉ}

Étudiante en Master 2 Intelligence et Innovation Culturelles - Université Paris Diderot, Assistante culture \& communication à la Fondation des Etats-Unis 